\title{
POSSIBILITIES AND THERAPY LIMITATIONS IN A CASE OF ADRENOGENITAL SYNDROME
}

\author{
Alina Grama ${ }^{1}$, Oana Marginean ${ }^{1}$, Lenuta Popa ${ }^{2}$, Lorena Elena Melit ${ }^{1}$, \\ Alexandra Latcu ${ }^{3}$, Mihaela Chincesan ${ }^{1}$ \\ ${ }^{1}$ Pediatrics Department I, University of Medicine and Pharmacy, Targu-Mures \\ ${ }^{2}$ Pediatrics Department I, University of Medicine and Pharmacy, Cluj Napoca \\ ${ }^{3}$ Clinical County Hospital, Targu-Mures
}

\begin{abstract}
Introduction. The adrenogenital syndrome with neonatal onset can manifest by repeated vomiting, dehydration and severe hydro-electrolytic disequilibria. The diagnostic approach, the metabolic reequilibration and the maintenance of the electrolytic balance in normal ranges by chronic treatment can be associated with difficulties.

Objective. We present a case of adrenogenital syndrome with severe neonatal onset, evolution accompanied by different complications, but with a prognosis improved by the adequate chronic treatment.

Material and method. A male child with small birth weight, was admitted in the Pediatrcs Clinic I, Targu-Mures, at the age of 3 weeks, with a severe clinic-biological syndrome by chronic vomiting and severe dehydration, and apparent macrogenitosomia. We performed a wide spectrum of investigations in acute phase, but also afterwards for the elaboration of the differential diagnosis.

Results. We identified persistent hyponatremia and hypopotassemia, an increased level of 17-OH progesterone $(44.96 \mathrm{ng} / \mathrm{ml})$ and the cortisol under the normal limit $(3 \mathrm{ug} / \mathrm{dl})$. We discovered significantly increased concentrations of ACTH (103 pg/ml in the morning) and total serum cholesterol $(186.72 \mathrm{ng} / \mathrm{dl})$. The macromolecular analysis pointed out the heterozygote genotype for the following mutations: 12G, R356W, P453S. The adequate chronic treatment with cortisone improved the prognosis; at the age of 1 years and 5 months, he presents an adequate height, weight and psychomotor development.

Conclusions. We fulfilled the conditions of the diagnosis of adrenogenital syndrome with neonatal onset in a heterozygote child. The small age the uncharacteristic symptomatology can be a burden for establishing an early diagnosis of adrenogenital syndrome.
\end{abstract}

Keywords: vomiting, newborn, hyponatremia

\section{INTRODUCTION}

Vomiting are among the most frequent symptoms present in the pediatric age, being the consequence of some infectious or metabolic disorders, obstructions at different levels of the digestive tract, neuropsychiatric disorders or they can appear after the intake of some toxic substances.

The congenital adrenal hyperplasia the adrenogenital syndrome represents a group of autosomal recessive transmission disorders caused by the deficit of one or multiple enzymes involved in the normal synthesis of the steroids from the three hormonal main classes: mineralocorticoids (aldosterone), glucocorticoids (cortisol) and sexual hormones. Taking under consideration the severity of the clinical manifestations, the adrenogenital syndrome can be divided in a classical form (severe, with neonatal onset) divided in the variant associated with salt loss and in that without loss of salt, and a non-classical form (criptogenetic, with late onset) (1). The diagnosis of adrenogenital syndrome by deficit of 21-hydroxilase is established through the quantitative determination of the steroid hormones and their precursors $(2,3)$.

\section{CASE PRESENTATION}

We present the case of a patient with persistent vomiting and severe dehydration even since the neonatal period, admitted in the Pediatrics Clinic 1, Targu-Mures, at the age of 26 days. From the heredo-collateral antecedents, we mention the lactose intolerance in one of the family members, the mother with obesity and uninvestigated hirsutism.

Corresponding author:

Oana Marginean, University of Medicine and Pharmacy, 38 Gh. Marinescu Street, Targu-Mures

E-mail: marginean.oana@gmail.com 
The personal physiological history: born at term, by cesarean section for pelvic presentation, with a weight of $2,550 \mathrm{~g}$ and a length of $52 \mathrm{~cm}$, APGAR score of 9, breastfed for 2 weeks, afterwards with formula, with weight stagnation.

History of the disease the onset of the disease is since the moment of birth with post-alimentation frequent vomiting, some in jet and unsatisfactory weight gain.

At the moment of admission, the clinical exam reveals a newborn with influenced general status, weight deficit approximately $600 \mathrm{~g}$, without fever, sorrowful facies, pallor, persistent skinfold, diminished cutaneous turgor, "café au lait" spot on the right limb, very weak represented adipose tissue, anterior fontanelle of $3 / 3 \mathrm{~cm}$, depressed, cardio-respiratory equilibrated, apparent macrogenitosomia.

\section{THE LABORATORY TESTS}

The laboratory tests do not show any acute infection at the moment of admission (CBC, peripheral smear, inflammatory tests in normal limits, urine culture and stool culture without any pathological modifications), thoracic radiography without congestive signs, without active pleural or pulmonary lesions, and the cardiologic exam does not reveal any significant modifications. The ionogram performed at the moment of admission reveals severe hyponatremia ( $\mathrm{Na} 117 \mathrm{mmol} / \mathrm{l})$ with hyperpotassemia (K $6.3 \mathrm{mmol} / \mathrm{l})$. The hepatic tests are modified (GOT 138.3 U/1, GPT 147,8 U/1, gamaCT 1529 U/l) with normal values of bilirubin. The patient presented at admission asymptomatic hypoglycemia (glycaemia $35 \mathrm{mg} \%$ ). All these modifications were initially interpreted as hydro-electrolytic disequilibria in context of an acute dehydration syndrome in a newborn with repeated vomiting and hepatic cholestasis.

Other investigations: abdominal radiography reveals severe bloating, but without pneumoperitoneum, without hydro-aired levels. The barium esogastro-duodenal passage excludes an antro-pyloric stenosis, a hiatal hernia or a gastroesophageal reflux. The hepatic serology is negative, the viral markers of TORCH syndrome are non-reactive. The immunogram is normal.

We raised the suspicion of lactose intolerance, taking under consideration the family history, we performed a genetic test that revealed a $\mathrm{C} / \mathrm{C}$ genotype of the 13,910 position and a G/G genotype of the 22,018 position (associated with the decreasing of the enzymatic activity of lactase). We initiated a diet with formula without lactose, general mea- sures: thermic comfort, the permeabilization of the airways, a good oxygenation, a way of peripheral approach, maintaining the optimal ventilation, monitoring the cardiac activity, checking the weight curve, checking the liquid intake, monitoring the diuresis. We performed hydro-electrolytic reequilibration with glucose and electrolytes, 10\% aminoacids, liver and gastric protectors by vein, probiotics. Under the administered treatment, the frequency of the vomiting diminished, but the hydro-electrolytic disequilibrium persisted (hyponatremia and hyperpotassemia), reason for which, we checked the way of taking the blood tests in order to avoid the wrong results of the ionogram and we started the EKG monitoring. We performed hormone tests. We discovered an increased value of the $17-\mathrm{OH}$ progesterone: $44.96 \mathrm{ng} / \mathrm{ml}$ (NV 3.6-13.7 ng/ml). The values of the thyroid-stimulating hormone (TSH) were constantly in normal limits, but the value of cortisol was under the normal limit: $3 \mathrm{ug} /$ dl (NV 4.3-22 ug/dl). The endocrinologic consult confirms clinically the adrenogenital syndrome (form with loss of salt) and recommends hydro-saline reequilibration, introduction of glucocorticoids by vein in bolus - Hemisuccinate hydrocortisone $25 \mathrm{mg}$, then $2 \times 12.5 \mathrm{mg}$, and afterwards $15-25 \mathrm{mg} /$ $\mathrm{m}^{2}$ divided in 3 doses plus saline intake orally through diet associated with mineralocortoids Fludrocortisone $0.15 \mathrm{mg} / \mathrm{m}^{2} /$ day orally.

\section{EVOLUTION}

The evolution was unpredictable regarding both, the symptomatology and complications, he presented after 3 weeks, systemic infection with Staphylococcus aureus MRSA for which he received antibiotic and supportive treatment with favorable evolution. The treatment was administered on central venous catheter since the first day of admission. The interpretation of the result was burdened by the small onset age and the impossibility of genetic confirmation in our clinic, reason for which the patient is transferred in the Pediatrics Clinic I, Cluj-Napoca, the Department of Pediatrics Endocrinology and Medical Genetics for confirmation of the genetic diagnosis and the establishment of the further therapeutic approach.

\section{THE POSITIVE AND DIFFERENTIAL DIAGNOSIS}

We discovered normal concentrations of the DHEA-S, significantly increased for ACTH: 103 $\mathrm{pg} / \mathrm{ml}$ in the morning (NV: 7-63 pg/ml) and for the 
total serum testosterone: $186.72 \mathrm{ng} / \mathrm{dl}$. The molecular analysis indicated in the patient the heterozygote genotype for the following mutations: $12 \mathrm{G}$, R356W, P453S. Afterwards, it was identified the maternal heterozygote genotype $12 \mathrm{G}$, and the paternal heterozygote genotype $\mathrm{R} 356 \mathrm{~W}$, respectively, important aspect for the family counseling.

There were excluded the following causes of regurgitations: regurgitation by the wrong technique of alimentation, the gastroesophageal reflux, achalasia, atresia, duplication and congenital esophagus stenosis, hiatal hernia, anomalies of the aortic arc.

There were taken under consideration the vomiting of digestive origin in the neonatal period: intestinal atresia, anatomic obstacles, necrotizing enterocolitis, diaphragmatic hernia, Hirschsprung neonatal disease, but also the etradigestive vomiting: infectious pathology (ENT infections, urinary tract infections, meningitis, respiratory infections, acute diarrheic diseases, sepsis), metabolic disorders (organic acidurias, phenylketonuria).

We excluded the false hyperpotassemia through wrong techniques of testing, exogenous and endogenous overloading of potassium, impaired renal excretion (acute and chronic renal insufficiency), deficit of mineralocorticoids (Adisson disease, enzymatic deficiencies), insufficient secretion of renin (diabetes mellitus). It was also excluded the hyponatremia through excessive loss of $\mathrm{NaCl}$ from the diarrheic syndrome, cystic fibrosis, thermic shock, adrenal insufficiency, through excessive intake of water (acute renal insufficiency, therapeutic errors, coma).

\section{DISCUSSIONS}

The adrenogenital syndrome is a genetic disorder that affects the steroids synthesis of the adrenal gland. The deficit of 21-hydroxilase is encountered

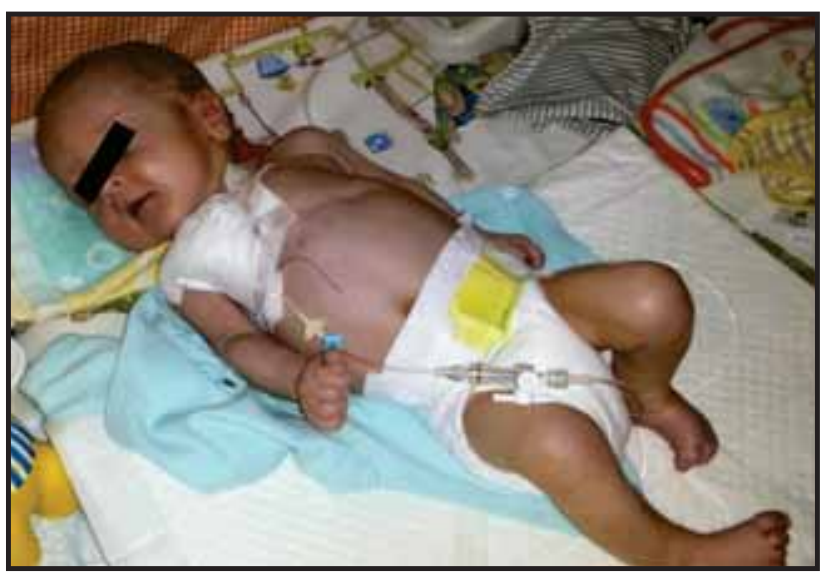

in $90 \%$ of the cases, the fundamental pathogenic element being the partial or total blockage of hydroxylation in the $21^{\text {st }}$ position. As a result, the hypophysis secretion of ACTH and cortisol precursors (17-OH-progesterone) is increased which leads to androgens excess $(4,5)$. In the classical form of adrenal congenital hyperplasia which appears in male newborns with normal external genital organs, the loss of salt represents a sign of great probability.

There were described until now over $100 C Y$ P21A2 mutations (functional gene for adrenal 21-hydroxilase), the identification of combined homozygote or heterozygote status confirming the diagnosis of classical deficit of 21-hydroxilase. The majority of the affected children's parents are heterozygote, clinically asymptomatic, but they can present mildly increased values of $17-\mathrm{OH}$ progesterone during the stimulation with ACTH. It is important that both parents are tested because in some cases a parent can be identified to have a non-classical form of disease.

The treatment concerns the prevention of salt loss and of vascular collapse if these exist, the substitution hormonal treatment for the substitution of cortisol and aldosterone secretion. The suppressive therapy is used with orally hydrocortisone in dose of $20 \mathrm{mg} / \mathrm{m}^{2} /$ day. For the substitution with mineralo-regulating hormones, the best is fludocortisone $0.15 \mathrm{mg} / \mathrm{m}^{2} /$ day orally. The salt supliment of $2-4$ grams/day is an useful adjuvant in the cases with salt loss 6).

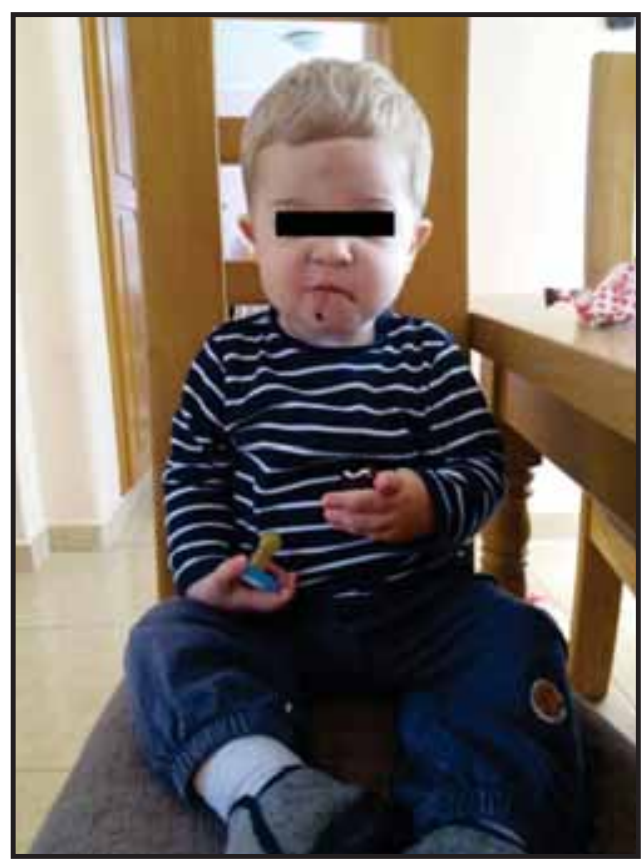

FIGURES 1, 2. Patient's evolution at the age of 3 weeks and 1.5 years, respectively 
General complications can be present (adrenal crisis with hyponatremia and shock, the early development of secondary sexual characters of the gluocorticoids overdosage), of hyponatremia (cerebral edema, pulmonary edema, prerenal isufficiency, severe dehydration) and of complications due to hyperpotassemia (heart stop in diastole, hyperpotassiemic paralysis) (7). In the well treated cases, the evolution is satisfactory, the final stature is at the inferior limit of normal, and the fertility is reestablished. The social and professional insertion of the patients is difficult in both genders $(8,9)$. In cases that suffered repeated reanimations in the first months of life, there can exist neurological sequelae, and the intelligence coefficient can be decreased.

\section{REFERENCES}

1. Witchel S.F. Nonclassic congenital adrenal hyperplasia. Curr Opin Endocrinol Diabetes Obes 2012; 19(3):151-8

2. Ambroziak U., Bednarczuk T., Ginalska-Malinowska M. et al. Congenital adrenal hyperplasia due to 21-hydroxylase deficiency management in adults. Endokrynol Pol 2010; 61(1):142-55

3. Falhammar H., Nyström H.F., Ekström U. et al. Fertility, sexuality and testicular adrenal rest tumors in adult males with congenital adrenal hyperplasiam. Eur J Endocrinol 2012; 166(3):441-9

4. Merke D.P. Approach to the adult with congenital adrenal hyperplasia due to 21-hydroxylase deficiency. J Clin Endocrinol Metab 2008; 93(3):653-60

5. Claahsen-van der Grinten H.L., Otten B.J., Hermus A.R., Sweep F.C., Hulsbergen-van de Kaa C.A. Testicular adrenal rest tumors in patients with congenital adrenal hyperplasia can cause severe testicular damage. Fertil Steril 2008; 89:597-601

6. German A., Suraiya S., Tenenbaum-Rakover Y., Koren I., Pillar G., Hochberg Z. Control of childhood congenital adrenal hyperplasia and
The malignisation can be present in adrenogenital syndrome. There were described also benign testicular tumors that recurred after the correct treatment of the disease $(10,11)$.

\section{CONCLUSIONS}

The early establishment of the adrenogenital syndroe diagnosis is essential in all the cases and the continuation of the treatment is important in order to reach a normal height and reproductive maturation, for obtaining a pshycho-intellectual optimal development. The prophylaxis of the disease includes the detection of the heterozygotes and the genetic advice.

sleep sctivity and quality with morning or evening glucocorticoid therapy. J Clin Endocrinol Metab 2008; 93(12):4707-4710

7. Rey C., Los-Arcos M., Hernández A. et al. Hypotonic versus isotonic maintenance fluids in critically ill children: a multicenter prospective randomized study. Acta Paediatr 2011; 100(8):1138-43

8. Falhammar H., Thoren M. Clinical outcomes in the management of congenital adrenal hyperplasia. Endocrine 2012; 41(3):355-373

9. Hindmarsh P.C. The child with difficult to control congenital adrenal hyperplasia. Is there a place for continuous subcutaneous hydrocortisone therapy. Clin Endocrinol 2014; 81(1):15-18

10. Chiriță A., Cojocaru R., Marazan M. et al. Tumori adrenotesticulare la un adolescent cu sindrom adrenogenital - rezultate după un an. Ro J Pediatr 2012; 1:69-76

11. Claahsen-van der Grinten H.L., Sweep FCGJ, Blickman J.G., Hermus ARMM, Otten B.J. Prevalence of testicular adrenal rest tumours in male children with congenital adrenal hyperplasia due to 21-hydroxylase deficiency. Eur J Endocrinol 2007; 157(3):339-344 